Journal of Animal and Veterinary Advances 11 (4): 443-448, 2012

ISSN: $1680-5593$

(C) Medwell Journals, 2012

\title{
Differential Neurotrophin-4 mRNA Expression in Reproductive Organs of Prepubertal Gilts with Different Dietary Energy Level
}

\author{
${ }^{1}$ Lina Tang, ${ }^{1} \mathrm{Lu}$ Chen, ${ }^{1}$ Chunjin Li, ${ }^{1,2}$ Yongfeng Sun, ${ }^{1}$ Yanling Sun and ${ }^{1} \mathrm{Xu}$ Zhou \\ ${ }^{1}$ Jilin Provincial Key Laboratory of Animal Embryo Engineering, \\ College of Animal Science and Veterinary Medicine, \\ Jilin University, 130062 Changchun, P.R. China \\ ${ }^{2}$ College of Animal Science and Technology, \\ Jilin Agricultural University, 130118 Changchun, P.R. China
}

\begin{abstract}
The development of reproductive system in prepubertal gilts was affected not only by gonadotropins but also by other cytokines and growth factors. Recent studies have revealed that Neurotrophins (NTs) which are well known for their essential roles in neural system also expressed in mammalian reproductive organs and involved in the regulation of mammalian reproduction. Dietary energy level is an important factor affecting domestic animal reproductive performance including central effects on Hypothalamic Pituitary Gonadal (HPG) axis and local effects on gonads. In the present study, the expression of Neurotrophin-4 (NT-4) which is a member of NTs in reproductive tissues of prepubertal gilts including ovary, oviduct and uterus was studied by immunohistochemistry. The effects of dietary energy on NT-4 mRNA expression were also investigated by quantitative PCR. The results show that NT-4 was localized in all classes of follicles in ovaries of prepubertal gilts including primordial follicles, primary follicles and secondary follicles, NT-4 was localized in mucosal epithelial cells in oviducts and glandular epithelium and endometrial epithelium in uteri. Furthermore, different dietary energy levels altered mRNA expression level of NT-4 in ovaries, uteri and oviducts of prepubertal gilts. NT-4 mRNA expression level in ovaries and uteri was higher in high dietary energy group than that in mid and low-energy groups. In oviduct, the expression pattern of NT-4 mRNA among three different dietary energy groups is similar as that occurred in in ovaries and uteri but with more significant differences. These results suggest that NT-4 may be one of the signals that links metabolic status and neuroendocrine control of reproduction in pig.
\end{abstract}

Key words: Prepubertal gilts, NT-4 mRNA, Hypothalamic Pituitary Gonadal (HPG), gonads, Neurotrophins (NTs), follicles, oviducts

\section{INTRODUCTION}

The reproductive performance of female animals is regulated by sophisticated mechanisms including endocrine regulation through HPG axis and local regulation in gonads. Numerous cytokines and growth factors have been documented in regulating the development and function of female reproductive system. NTs including Nerve Growth Factor (NGF), Brain-Derived Neurotropic Factor (BDNF), Neurotrophin-3 (NT-3) and Neurotrophin-4 (NT-4 also known as NT-5) have been well-characterized for their critical roles in central and peripheral nervous systems (Jones et al., 1994; Barbacid, 1995).

In the past few years, increasing numbers of studies have shown that several NTs and their receptors are also expressed in the mammalian reproductive organs and involved in the endocrine hormonal mechanisms controlling ovarian development, primordial follicle formation, early follicular development, ovulation, oocytes maturation and embryonic development in several species (Dissen et al., 1996; Yi et al., 2008; Zhang et al., 2010; Anderson et al., 2010).

Energy intake can also affect female reproductive function by metabolic control that alters hypothalamic Gonadotropin-Releasing Hormone $(\mathrm{GnRH}) /$ Luteinizing Hormone (LH) pulse, impacts secretion of follicular steroid hormones and alters ovarian sensitivity to gonadotropin and cytokines (Temple and Rissman, 2000; Almeida et al., 2001; Barb et al., 2004, 2005; Webb et al., 2004). Recent findings demonstrate that numerous genes i.e., relaxin, interleukins and other cytokines as well as biologically

Corresponding Author: Xu Zhou, Jilin Provincial Key Laboratory of Animal Embryo Engineering, College of Animal Science and Veterinary Medicine, Jilin University, 130062 Changchun, P.R. China 
active substances such as leptin, Insulin-like Growth Factor-I (IGF-I), IGF-II and agouti protein could have a profound effect on metabolic status and the reproductive axis (Barb et al., 2005). It is of great relevance and significance to better understand the function of neurotrophin-4 in regulating the development of the reproductive system of prepubertal gilts especially how this function could be manipulated through the alteration of dietary energy level thus to raise gilts to have better reproductive performance when mature. In the present study, the expression of NT-4 in reproductive tissues of prepubertal gilts was examined by immunohistochemistry and the effects of dietary energy level on NT-4 mRNA expression were investigated by quantitative PCR.

\section{MATERIALS AND METHODS}

Ethics statement: All experimental procedures in the present research were approved by the Animal Care and Use Committee of Jilin University.

Immunohistochemistry: The ovaries, oviducts and uteri of prepubertal gilts were obtained from a public slaughter house. The samples were collected within 15 min after slaughter and then fixed with $2 \%$ paraformaldehyde for $24 \mathrm{~h}$. After embedded in paraffin, $5 \mu \mathrm{m}$ thick sections were cut following a standard procedure. Slides were first incubated in three washes of xylene for $5 \mathrm{~min}$ each and then incubated in two washes of $100 \%$ ethanol for $10 \mathrm{~min}$ each followed by two washes of $95 \%$ ethanol for $10 \mathrm{~min}$ each. After final two washes in $\mathrm{ddH}_{2} \mathrm{O}$, slides were first incubated in $3 \%$ hydrogen peroxide for $10 \mathrm{~min}$ and then blocked with normal goat serum (10\%) in PBS for $30 \mathrm{~min}$ at room temperature. Slides were incubated with a primary antibody (anti-NT-4, 1:100, BOSTER, China) at $4^{\circ} \mathrm{C}$ for 36 h. Following several washes with PBS, slides were incubated with peroxidase conjugated goat anti-rabbit IgG (1:200; BOSTER, China) for $30 \mathrm{~min}$ at room temperature. After washing with PBS, slides were incubated with DAB substrate for color development. Negative controls were prepared using rabbit IgG instead of primary antibody diluted with PBS. Finally, the slides were examined under an optical microscope (Olympus, Japan). Sections of each sample were examined in triplicates for both positive antibody staining and negative controls.

Animals, dietary, feeding and management: Healthy prepubertal crossbred (Landrace $x$ Yorkshire) gilts at the age of 60 days and body weight of $15 \mathrm{~kg}$ were used in this study. Eighteen gilts were randomly divided into three groups $(n=6)$. Gilts in the mid-energy group (group $M$ ) were fed with diets designed according to the NRC (1998) standard of USA with energy intake meeting $100 \%$ of
Table 1: Ingredient and nutrient composition of diets feed to prepubertal gilts in different groups

\begin{tabular}{lrrr}
\hline Ingredient and nutrient composition & \multicolumn{1}{c}{$\mathrm{HE}$} & \multicolumn{1}{c}{$\mathrm{ME}$} & \multicolumn{1}{c}{$\mathrm{LE}$} \\
\hline Com (\%) & 34.400 & 50.400 & 46.500 \\
Wheat (\%) & - & 3.200 & 22.000 \\
Soybean meal (\%) & 13.900 & 20.600 & 20.700 \\
Extruded soy powder (\%) & 18.000 & 8.000 & 2.000 \\
DDGS (\%) & 10.000 & 6.000 & 4.000 \\
Sucrose (\%) & 5.000 & 4.000 & 1.000 \\
Premix (\%) & 1.000 & 1.000 & 1.000 \\
Soybean oil (\%) & 5.000 & 1.000 & - \\
Salt (\%) & 0.080 & 0.080 & 0.060 \\
Com starch (\%) & 10.800 & 4.000 & 1.000 \\
Stone powder (\%) & 0.840 & 0.900 & 1.180 \\
Calcium bicarbonate (\%) & 0.900 & 0.720 & 0.400 \\
Lysine (\%) & 0.080 & 0.100 & 0.160 \\
Total energy & 16580.000 & 14937.00 & 13518.000 \\
(kJ kg ${ }^{-1}$, assayed) & & & \\
Digestive energy & 15727.000 & 14254.000 & 12849.000 \\
(kJ kg ${ }^{-1}$, calculated) & & & \\
Crude protein (\%) & 18.000 & 18.000 & 18.000 \\
Calcium (\%) & 0.620 & 0.600 & 0.640 \\
Bioavailable phosphorus (\%) & 0.290 & 0.260 & 0.230 \\
Total phosphorus (\%) & 0.500 & 0.500 & 0.560 \\
Salt (\%) & 0.110 & 0.120 & 0.130 \\
Lysine (\%) & 0.964 & 0.950 & 0.951 \\
Methionine (\%) & 0.307 & 0.306 & 0.281 \\
Threonine (\%) & 0.721 & 0.727 & 0.689 \\
\hline About & kg $1 \%$ pro
\end{tabular}

About $1 \mathrm{~kg}$ of $1 \%$ premix consists of iodine $14 \mathrm{mg}$, manganese $300 \mathrm{mg}$, zincum $8000 \mathrm{mg}$, copper $500 \mathrm{mg}$, iron $8000 \mathrm{mg}$, selenium $25 \mathrm{mg}$, VA $175000 \mathrm{IU}$, VD3 $20000 \mathrm{IU}$, VE $1100 \mathrm{IU}$, VK $50 \mathrm{mg}$, biotin $5 \mathrm{mg}$, choline $40000 \mathrm{mg}$, folic acid $30 \mathrm{mg}$, nicotinic acid $1250 \mathrm{mg}$, pantothenic acid $900 \mathrm{mg}$, riboflavin $300 \mathrm{mg}$, thiamine $100 \mathrm{mg}$, VB6 $150 \mathrm{mg}$, VB12 $1500 \mu \mathrm{g}$

digestive energy requirement. Gilts in the low energy group (group L) were fed with diets with an energy level $10 \%$ lower than that in the standard diet with energy intake meeting $90 \%$ of digestive energy requirement. Gilts in the high energy group (group H) were fed with diets with an energy level 10\% higher than that in the standard diet with energy intake meeting $110 \%$ of digestive energy requirement (ingredient and nutrient composition as shown in Table 1). All gilts were fed 3 times daily ad libitum at 6:00 am, 11:00 am and 6:00 pm with water freely available.

Gilts were housed within pens in an enclosed building with constant temperature $\left(21 \pm 3^{\circ} \mathrm{C}\right)$ and relative humidity $(55 \pm 3 \%)$, under controlled lighting conditions. Feeding experiments were lasted for 12 days. At the end of the feeding experiments, ovaries, oviducts and uteri were recovered and kept in liquid nitrogen until transferred into $-80^{\circ} \mathrm{C}$ refrigerator.

RNA extraction and cDNA synthesis: Total RNA was extracted from the samples stored in $-80^{\circ} \mathrm{C}$ refrigerator using Trizol reagent (Invitrogen Life Technologies Inc., USA) and then treated with Rnase-free Dnase I (Tiagen) in order to eliminate genomic DNA. The concentration of RNA samples were determined with a spectrophotometer (SANYO, Japan) and then diluted into equal 
concentrations with Rnase-free distilled water. Oligo $(\mathrm{dT})_{18}$ was used as primer for reverse transcription and the cDNA samples were frozen at $-20^{\circ} \mathrm{C}$ until use.

Quantitative real-time PCR: Real-time PCR was carried out in an ABI PRISM 7000 machine according to the manufacturer's instructions. For amplification, reactions were performed in a $25 \mu \mathrm{L}$ final volume containing $100 \mathrm{ng}$ of cDNA reverse transcribed from mRNA samples of uteri, ovaries and oviducts of prepubertal gilts in different feed energy level groups, $0.15 \mu \mathrm{L}$ of Ex Taq HS (TakaRa), $2.5 \mu \mathrm{L}$ of $10 \times \mathrm{Ex}$ Taq Buffer (TakaRa), $2.0 \mu \mathrm{L}$ of dNTP mixture $(2.5 \mathrm{mmol} / \mathrm{L} / \mathrm{each}$ ) (TakaRa), $0.5 \mu \mathrm{L}$ of primers $\left(10 \mu \mathrm{mol} \mathrm{L}^{-1}\right), 0.25 \mu \mathrm{L}$ of TAMRA-FAM $\left(10 \mu \mathrm{mol} \mathrm{L}^{-1}\right)$ and distilled water to $25 \mu \mathrm{L}$. The PCR reaction was composed of one cycle of $6 \mathrm{~min}$ at $95^{\circ} \mathrm{C}$ followed by 48 cycles of $30 \mathrm{sec}$ at $94^{\circ} \mathrm{C}, 30 \mathrm{sec}$ at $59^{\circ} \mathrm{C}$. A standard curve was generated and used to evaluate the relative expression of the NT-4 gene in terms of the ratio (fold difference) of the target gene expression over the control gene expression. The specific primers and probes used for NT-4 were NT-4FP: 5'-AGTCCTACGTGCGGGCATT-3', NT-4-RP: 5'CACAGGCAGTGTCAATTCGAA- ${ }^{\prime}$ andNT-4-TAMRAFAM: 5'-CACCGATGCCCAGGGCCGT-3'.

Statistical analysis: The data are presented as mean $\pm \mathrm{SE}$. Comparisons between groups were performed by one-way ANOVA. The significance of differences between the mean values in each treated group was tested with Duncan's multiple-comparison test. A value of $p<0.05$ was considered statistically significant and a value of $\mathrm{p}<0.01$ was considered statistically highly significant.

\section{RESULTS}

Immunohistochemistry staining of NT-4 in the uteri, ovaries and oviducts of prepubertal gilts: As shown in Fig. 1, NT-4 was detected in ovaries, uterus and oviducts

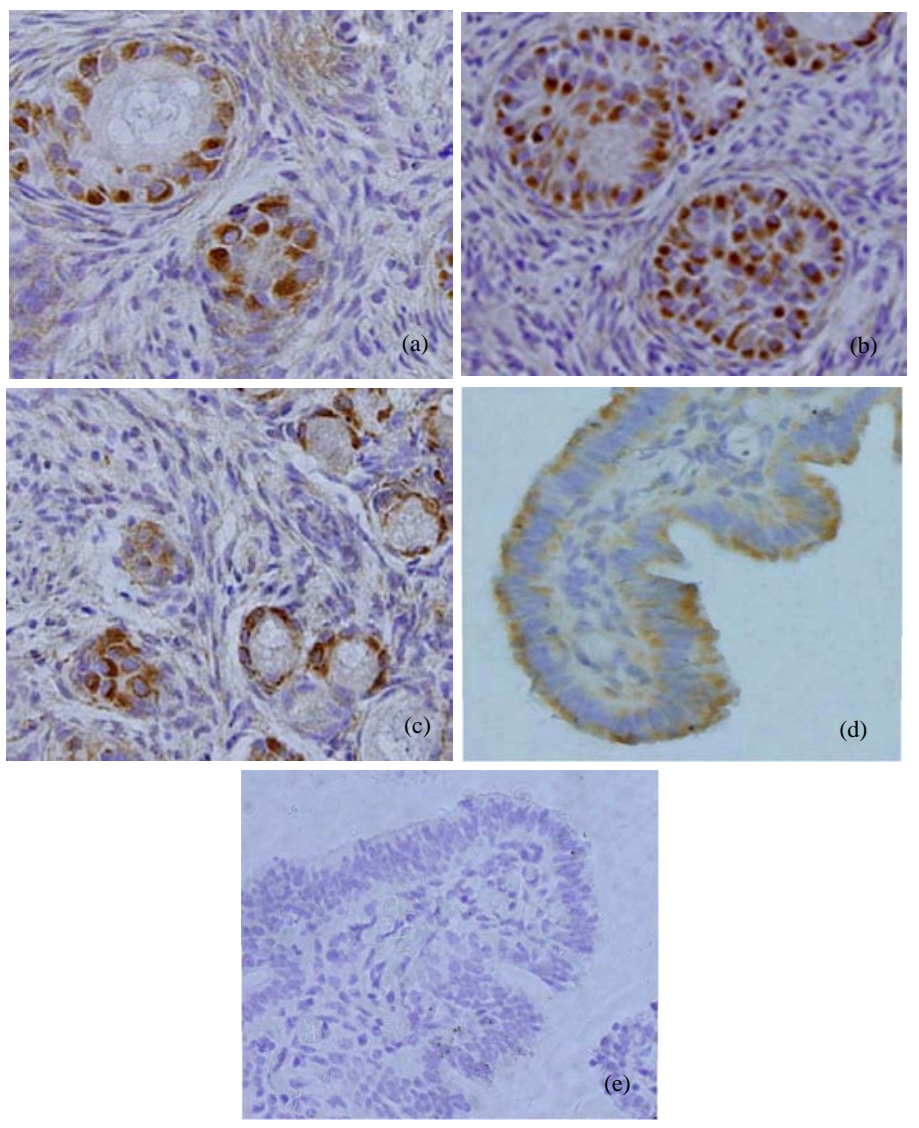

Fig. 1: Detection of the NT-4 in the prepubertal pig ovary, oviduct and uterus; a) NT-4 immunoreactivity is predominantly seen in primordial follicles, primary follicles; b) secondary follicles; c) NT-4 is detected in the uterine glandular epithelium and endometrial epithelium; d) NT-4 immunoreactivity is predominantly seen in oviduct mucosal epithelial cells and e) section incubated with rabbit IgG instead of primary antibody served as negative control. a, c, d, e: original magnification x100. c: original magnification $\times 40$ 


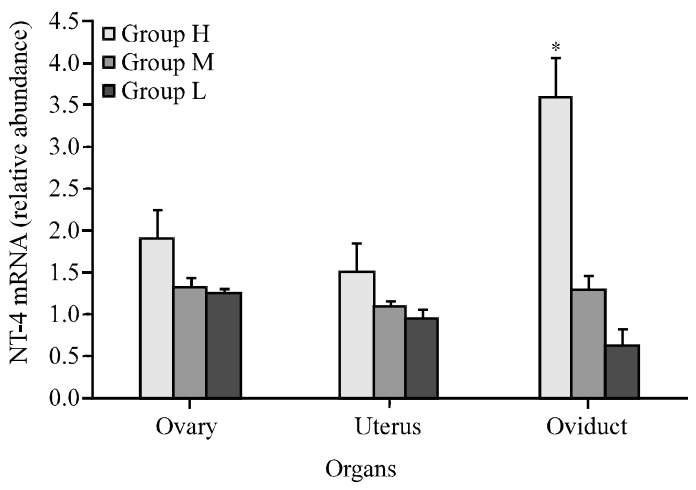

Fig. 2: Relative abundance (arbitrary units) of NT-4 mRNA in reproductive tissues of prepubertal gilts in different energy intake group. ${ }^{*} \mathrm{p}<0.05$

of prepubertal gilts by immunohistochemical staining. In the ovary, NT-4 was detected in different classes of follicles including primordial follicles, primary follicles and secondary follicles. The strongest staining of NT-4 appeared to be in the granulosa cells. In the oviducts, positive staining was observed in the mucosal epithelial cells. In the uterus, NT-4 was localized in the uterine glandular epithelium and endometrial epithelium. Furthermore, a positive staining for NT-4 was seen in the myometrial vascular smooth muscle cells.

Real-time PCR analysis of NT-4 mRNA expression in the uteri, ovaries and oviducts of prepubertal gilts in different feed energy intake groups: As shown in Fig. 2, group $\mathrm{H}$ showed significant higher amounts of NT-4 mRNA expressions in all of the reproductive organs than that in group $M$ and $L(p<0.05)$. Among different energy intake groups, NT-4 mRNA expression in oviducts showed the most significant change affected by diet energy while in the uterus and ovaries, higher NT-4 mRNA expression was detected in group $H$ and $M$ than in group $\mathrm{L}$ but the difference was not significant $(\mathrm{p}>0.05)$. NT-4 mRNA expression showed variations among different tissues in the same energy intake groups. In the high diet energy group, NT-4 mRNA expression in oviducts was significantly higher than that in ovaries and uterus $(p<0.05)$. However, in mid-energy group and low-energy group, there were no significant differences of NT-4 mRNA expression among different organs $(\mathrm{p}>0.05)$.

\section{DISCUSSION}

Dietary energy level is one of the most important factors that affect animal reproductive performance; it has significant impact on the development of reproductive system, maintenance of estrous cycle, mating, fertilization and embryo development. A number of studies have shown that energy intake restriction could impact on the
HPG axis of gilts and sows through the regulation of gonadotropin. It has been suggested that the responses of the gonads to nutritional/metabolic signaling could also participate in the modulation of mammalian reproduction (Cox et al., 1987; Cosgrove and Foxcroft, 1996; Lucy, 2008). In the last two decades, the role of NTs in regulating female and male reproductive performance has been clearly established (Dissen et al., 2001; Tometten et al., 2005; Sun et al., 2011). However, the alteration of NT-4 mRNA expression level impacted by energy intake level is still unclear. To the knowledge, this is the first study to research the NT-4 expression status in different dietary energy intake levels in prepubertal gilts reproductive system.

Regulation of ovarian activity is an integrated process encompassing both extraovarian signals and intrafollicular factors (Webb et al., 2004). In mouse, NT-4 expressed in oocytes and in human, NT-4 expressed in granulosa cells at the early developmental stages of epithelioid cells. NT-4 could promote primary oocyte development and the maturation of granulosa cells (Childs et al., 2010). In the present study, NT-4 was shown to be localized in all classes of follicles in prepubertal gilts ovaries including primary follicles, secondary follicles and even in the primordial follicles. This result suggests that NT-4 may be one of the key members involved in porcine granulosa cell proliferation and follicular development in an autocrine or paracrine way. When gilts were fed with diets of different energy levels, NT-4 expression level in high energy group was higher than that in group $\mathrm{M}$ and $\mathrm{L}$, though no statistically significant difference was found. This result indicates that ovarian NT-4 expression may be regulated by the energy metabolism signal

The primary function of the endometrium is to provide appropriate environment for the growth and development of fertilized eggs. Endometrial glands secrete nutrient supplies and support the growth of early embryos. Previous studies have shown that BDNF and NGF immunolocalized in the uterine tract of rodents (Bjorling et al., 2002; Krizsan-Agbas et al., 2003) and NT-4 located in the bovine uterus (Sun et al., 2011) while the exact location of NT-4 in the prepubertal gilts is still unclear. In this study, we found that NT-4 mainly expressed in the endometrial epithelial cells which indicated that NT-4 may be involved in a paracrine or autocrine network to establish and maintain pregnancy and fetal development. Furthermore, NT-4 expression levels in different dietary energy level groups changed in a similar way as that occurred in ovary. Higher NT-4 expression was detected in the group $\mathrm{H}$ than that in group $\mathrm{M}$ and $\mathrm{L}$ without statistically significant differences. 
Oviduct is very important for fertilization and early development of eggs. Early mammalian embryo cleavage is conceived in the fallopian tubes and tubal fluid provides suitable environment for the fertilization, embryo transportation and early embryos development (Hunter, 1981). It has been reported that NT-4 was absent in human fallopian tubes (Anderson et al., 2002) while NT-4 was located in the oviduct of cow in different stage of estrous cycle (Sun et al., 2011). In this study, NT-4 was immunolocalized in the epithelial cells of oviduct in prepubertal gilts suggesting that NT-4 may play important roles in the development of oviduct in gilts before puberty such as participating in the oviductal mucosal epithelium proliferation, differentiation and development. Furthermore, NT-4 mRNA expression significantly increased in group $\mathrm{H}$ compared with group $\mathrm{M}$ and $\mathrm{L}$. In addition, NT-4 expression level in oviducts was significantly higher than that in ovaries and uteri among all of the different energy intake levels in prepubertal gilts. Hence, NT-4 may be a very important factor in nutrition/metabolism regulatory mechanisms on the gilts oviduct.

\section{CONCLUSION}

In this study, NT-4 was expressed in ovaries, oviducts and uterus of the prepubertal gilts. Dietary energy level plays a role in regulating NT-4 mRNA expression in organs. The nutrition/metabolism signal may be involved in the control of reproductive function by alter NT-4 expression in reproductive tissue. The research adds neurotrophins, specifically NT-4 to the list of growth factors in regulating growth, development and functions of prepubertal gilts reproductive system.

\section{ACKNOWLEDGEMENTS}

Researchers would like to thank Dr. Xianzhong Yu (Dept. of Biological Sciences, Clemson University, USA) for revising and valuable suggestions for improvement of the research. This research was supported by the State Key Development Program of Basic Research (973 Program) of China (No. 932004) National Natural Science Foundation of China (No. 30671511).

\section{REFERENCES}

Almeida, F.R., J. Mao, S. Novak, J.R. Cosgrove and G.R. Foxcroft, 2001. Effects of different patterns of feed restriction and insulin treatment during the luteal phase on reproductive, metabolic and endocrine parameters in cyclic gilts. J. Anim. Sci., 79: 200-212.
Anderson, R.A., L.L. Robinson, J. Brooks and N. Spears, 2002. Neurotropins and their receptors are expressed in the human fetal ovary. J. Clin. Endocrinol. Metab., 87: 890-897.

Anderson, R.A., R.A. Bayne, J. Gardner and P.A. de Sousa, 2010. Brain-derived neurotrophic factor is a regulator of human oocyte maturation and early embryo development. Fertil. Steril., 93: 1394-1406.

Barb, C.R., G.J. Hausman and K. Czaja, 2005. Leptin: A metabolic signal affecting central regulation of reproduction in the pig. Domest. Anim. Endocrin., 29: 186-192.

Barb, C.R., J.B. Barrett and R.R. Kraeling, 2004. Role of leptin in modulating the hypothalamic-pituitary axis and luteinizing hormone secretion in the prepuberal gilt. Domest. Anim. Endocrinol., 26: 201-214.

Barbacid, M., 1995. Neurotrophic factors and their receptors. Curr. Opin. Cell Biol., 7: 148-155.

Bjorling, D.E., M. Beckman, M.K. Clayton and Z.Y. Wang, 2002. Modulation of nerve growth factor in peripheral organs by estrogen and progesterone. Neuroscience, 110: 155-167.

Childs, A.J., R.A.L. Bayne, A.A. Murray, S.J.M. Da Silva, C.S. Collins, N. Spears and R.A. Anderson, 2010. Differential expression and regulation by activin of the neurotrophins BDNF and NT4 during human and mouse ovarian development. Dev. Dynam., 239: 1211-1219.

Cosgrove, J.R. and G.R. Foxcroft, 1996. Nutrition and reproduction in the pig: Ovarian aetiology. Anim. Reprod. Sci., 42: 131-141.

Cox, N.M., M.J. Stuart, T.G. Althen, W.A. Bennett and H.W. Miller, 1987. Enhancement of ovulation rate in gilts by increasing dietary energy and administering insulin during follicular growth. J. Anim. Sci., 64: 507-516.

Dissen, G.A., C. Romero, A.N. Hirshfield and S.R. Ojeda, 2001. Nerve growth factor is required for early follicular development in the mammalian ovary. Endocrinology, 142: 2078-2086.

Dissen, G.A., D.F. Hill, M.E. Costa, C.W.L. Dees, H.E. Lara and S.R. Ojeda, 1996. A role for trkA nerve growth factor receptors in mammalian ovulation. Endocrinology, 137: 198-209.

Hunter, R.H., 1981. Sperm transport and reservoirs in the pig oviduct in relation to the time of ovulation. J. Reprod. Fertil., 63: 109-117.

Jones, K.R., I. Farinas, C. Backus and L.F. Reichardt, 1994. Targeted disruption of the BDNF gene perturbs brain and sensory neuron development but not motor neuron development. Cell, 76: 989-999. 
Krizsan-Agbas, D., T. Pedchenko, W. Hasan and P.G. Smith, 2003. Oestrogen regulates sympathetic neurite outgrowth by modulating brain derived neurotrophic factor synthesis and release by the rodent uterus. Eur. J. Neurosci., 18: 2760-2768.

Lucy, M.C., 2008. Functional differences in the growth hormone and insulin-like growth factor axis in cattle and pigs: Implications for post-partum nutrition and reproduction. Reprod. Domest. Anim., 43: 31-39.

NRC, 1998. Nutrient Requirement of Swine. 10th Rev. Edn., National Academy Press, Washington DC., USA.

Sun, Y.F., C.J. Li, Y.L. Sun, L. Chen and Z. Liu et al., 2011. Expression of neurotrophin 4 and its receptor tyrosine kinase $\mathrm{B}$ in reproductive tissues during the follicular and luteal phases in cows. Asian-Aust. J. Anim. Sci., 24: 336-343.

Temple, J.L. and E.F. Rissman, 2000. Acute re-feeding reverses food restriction-induced hypothalamicpituitary-gonadal axis deficits. Biol. Reprod., 63: 1721-1726.
Tometten, M., S. Blois and P.C. Arck, 2005. Nerve growth factor in reproductive biology: Link between the immune, endocrine and nervous system? Chem. Immunol. Allergy, 89: $135-148$.

Webb, R., P.C. Garnsworthy, J.G. Gong and D.G. Armstrong, 2004. Control of follicular growth: Local interactions and nutritional influences. J. Anim. Sci., 82: E63-E74.

Yi, K.L., X. Zhou, D.S. Shi, H.H. Chen and Q.L. Qin et al., 2008. The mRNA expression of brain-derived neurotrophic factor in oocytes and embryos and its effects on the development of early embryos in cattle. Animal, 2: 1786-1794.

Zhang, L., Y. Liang, Y. Liu and C.L. Xiong, 2010. The role of brain-derived neurotrophic factor in mouse oocyte maturation in vitro involves activation of protein kinase B. Theriogenology, 73: 1096-1103. 